\title{
A Derivation of the Path-Integral Formalism of Quantum Mechanics from Probability Conservation along the Velocity Flows of Classical Particles
}

\author{
Frank Blume* \\ Department of Mathematics, John Brown University, 2000 W. University St., Siloam Springs, AR 72761, USA
}

Received: 2 Jul. 2013, Revised: 18 Jul. 2013, Accepted: 21 Jul. 2013

Published online: 1 Aug. 2013

\begin{abstract}
Denoting by $\underline{\mathrm{v}}$ the velocity flow of a classical particle that is subject to a potential $V$, we demonstrate that the path-integral formalism of non-relativistic quantum mechanics can be obtained by superimposing wave functions that are solutions of a wave equation which in turn directly corresponds to the probability conservation equation $\operatorname{div}\left(\psi^{*} \psi \underline{\mathrm{v}}\right)=0$.
\end{abstract}

Keywords: Path-Integral, Quantum Mechanics, Classical Particles

\section{Introduction}

In order to introduce the present paper's subject matter, we denote by $z_{y, t, x}$ the spatial coordinate of a classical particle of mass $m$ that moves in one spatial dimension in the force field $\underline{F}=-\nabla V$ and satisfies the boundary conditions $z_{y, t, x}(0)=y$ and $z_{y, t, x}(t)=x$. The restriction to one spatial dimension is permissible because the extension to three spatial dimensions is very easily accomplished. Given this definition of $z_{y, t, x}$, the corresponding 2-velocity flow $\underline{\mathrm{v}}_{y}$ is

$$
\underline{\mathrm{v}}_{y}(t, x)=\left(\begin{array}{c}
1 \\
v_{y}(t, x)
\end{array}\right)=\left(\begin{array}{c}
1 \\
z_{y, t, x}^{\prime}(t)
\end{array}\right)
$$

and the flow lines of $\underline{\mathrm{v}}_{y}$ are described by the parameterization

$$
c_{y, t, x}(s):=\left(\begin{array}{c}
s \\
z_{y, t, x}(s)
\end{array}\right) .
$$

Note: in order for $\underline{\mathrm{v}}_{y}$ to be well defined for all values $t$ that are less than or equal to some positive constant $T$, we will assume that the boundary conditions $z_{y, t, x}(0)=y$ and $z_{y, t, x}(t)=x$ uniquely determine $z_{y, t, x}$ for all $t \in(0, T)$. The necessity and plausibility of this assumption will become clear when we later consider, as an example, the special case of a harmonic oscillator potential.
Associated with each $\underline{\mathrm{v}}_{y}$ we imagine to be given a probability density function $\rho_{y}$ which is thought to specify how likely it is for the given classical particle to be found on one of the flow lines $c_{y, t, x}$. That is to say, $\rho_{y}$ is assumed to satisfy the following preservation equation:

$$
\operatorname{div}\left(\rho_{y} \underline{\mathrm{V}}_{y}\right)=0 .
$$

In order to link this equation to quantum mechanics, we further assume that we are given a wave function $\psi_{y}$ such that $\left|\psi_{y}\right|^{2}=\psi_{y}^{*} \psi_{y}=\rho_{y}$. Then, according to (2), it must be the case that

$$
\psi_{y}^{*} \partial_{\underline{\mathbf{V}}_{y}} \psi_{y}+\psi_{y} \partial_{\underline{\mathbf{V}}_{y}} \psi_{y}^{*}=\partial_{\underline{\mathbf{v}}_{y}}\left(\psi_{y}^{*} \psi_{y}\right)=-\psi_{y}^{*} \psi_{y} \operatorname{div} \underline{\mathbf{v}}_{y} .
$$

By inspection we find that this equation is satisfied if there exists a real-valued function $L_{y}$ such that $\psi_{y}$ is a solution of the wave equation

$$
\partial_{\underline{\mathbf{v}}_{y}} \psi_{y}=-\frac{\psi_{y}}{2} \operatorname{div} \underline{\mathbf{v}}_{y}+i L_{y} \psi_{y}
$$

The purpose of the present paper is to demonstrate that this equation, when employed as a generating equation for path-integral superpositions, yields a description of non-relativistic quantum mechanics that is fully equivalent to the wave-mechanical formalism that the

\footnotetext{
*Corresponding author e-mail: fblume@jbu.edu
} 
Schrödinger equation describes. As a note of caution we wish to add that the possibility of founding quantum physics on equation (4) is so elementary a claim that it is hard to imagine its validity to be as yet unknown. However, after examining a very substantial number of potential sources, the present author was unable to find any article or book in which the claim in question has been established or discussed. No research paper or ordinary textbook appears to make any reference to it, and a comprehensive work on path-integration like [3] does not contain it either (unless the present author somehow overlooked it). In the light of these failed attempts to locate a relevant literature source, the content of the present paper is here being offered-with considerable hesitation-as a provisional novelty.

Remark 1According to (3), we have

$$
\frac{\partial \rho_{y}}{\partial t}+v_{y} \frac{\partial \rho_{y}}{\partial x}=\partial_{\underline{\mathbf{v}}_{y}} \rho_{y}=-\rho_{y} \operatorname{div} \underline{\mathrm{v}}_{y}=-\rho_{y} \frac{\partial v_{y}}{\partial x},
$$

and therefore,

$$
\frac{\partial}{\partial t} \int_{-\infty}^{\infty} \rho_{y} d x=-\int_{-\infty}^{\infty} \frac{\partial\left(\rho_{y} v_{y}\right)}{\partial x} d x=\left.\frac{\partial\left(\rho_{y} v_{y}\right)}{\partial x}\right|_{-\infty} ^{\infty}=0 .
$$

This shows that equation (2) does indeed guarantee the preservation of the total probability represented by the space integral of $\rho_{y}$.

\section{Free Particles}

In order to establish the link to quantum mechanics, to which we alluded in the Introduction, we observe to begin with that the probability conservation equation (2) uniquely determines the future evolution of the density function $\rho_{y}$ from a given initial state at time $t_{0}>0$. For using the parameterization $c_{y, t, x}$ in (1), we may apply equation (2) to infer that

$$
\begin{aligned}
0 & =\left.\operatorname{div}\left(\rho_{y} \underline{\mathrm{v}}_{y}\right)\right|_{c_{y, t, x}(s)} \\
& =\left.\partial_{\underline{\mathbf{v}}_{y}} \rho_{y}\right|_{c_{y, t, x}(s)}+\rho_{y}\left(c_{y, t, x}(s)\right) \operatorname{div} \underline{\mathbf{v}}_{y}\left(c_{y, t, x}(s)\right) \\
& =\frac{d}{d s} \rho_{y}\left(c_{y, t, x}(s)\right)+\rho_{y}\left(c_{y, t, x}(s)\right) \operatorname{div} \underline{\mathbf{v}}_{y}\left(c_{y, t, x}(s)\right)
\end{aligned}
$$

and that, by implication,

$$
\rho_{y}\left(c_{y, t, x}(s)\right)=\rho_{y}\left(c_{y, t, x}\left(t_{0}\right)\right) e^{-\int_{t_{0}}^{s} \operatorname{div} \underline{\underline{V}}_{y}\left(c_{y, t, x}(\tau)\right) d \tau} .
$$

Considering the simple case of a free particle, we find that

$$
\underline{\mathrm{v}}_{y}(t, x)=\left(\begin{array}{c}
1 \\
(x-y) / t
\end{array}\right)
$$

and

$$
c_{y, t, x}(s)=\left(\begin{array}{c}
s \\
s(x-y) / t
\end{array}\right) .
$$

Denoting the initial probability density at $t_{0}$ by $g$, i.e.,

$$
\rho_{y}\left(t_{0}, x\right)=g(x)
$$

and observing that

$$
\operatorname{div} \underline{\mathrm{v}}_{y}=\frac{1}{t},
$$

equation (5) allows us to infer that

$$
\rho_{y}(t, x)=g\left(t_{0}(x-y) / t\right) e^{-\int_{t_{0}}^{t} 1 / \tau d \tau}=\frac{t_{0} g\left(t_{0}(x-y) / t\right)}{t} .
$$

A quick comparison-for the case $y=0$ - shows that this result is compatible with the standard description of a stationary free-particle wave in elementary quantum mechanics: a standing-wave solution of the free Schrödinger equation

$$
i \frac{\partial \psi}{\partial t}=-\frac{\hbar}{2 m} \frac{\partial^{2} \psi}{\partial x^{2}}
$$

that satisfies the Gaussian initial condition

$$
\psi\left(t_{0}, x\right)=\frac{e^{-x^{2} /\left(4 \sigma^{2}\right)}}{\sqrt[4]{2 \pi \sigma^{2}}}
$$

is $\psi(t, x)=\frac{e^{-x^{2} /\left(4 \sigma^{2}\left(1+i \hbar\left(t-t_{0}\right) /\left(2 m \sigma^{2}\right)\right)\right)}}{\sqrt[4]{2 \pi \sigma^{2}}\left(1+i \hbar\left(t-t_{0}\right) /\left(2 m \sigma^{2}\right)\right)^{1 / 2}}$ (see [2], p.30),
with

$$
\rho=|\psi|^{2}=\frac{e^{-x^{2} /\left(2 \sigma^{2}\left(1+\hbar^{2}\left(t-t_{0}\right)^{2} /\left(2 m \sigma^{2}\right)^{2}\right)\right)}}{\sqrt{2 \pi} \sigma\left(1+\hbar^{2}\left(t-t_{0}\right)^{2} /\left(2 m \sigma^{2}\right)^{2}\right)^{1 / 2}}
$$

as the corresponding probability density. Setting

$$
t_{0}:=\frac{2 m \sigma^{2}}{\hbar}
$$

yields

$$
\begin{aligned}
\rho & =\frac{e^{-x^{2} /\left(2 \sigma^{2}\left(1+\left(t-t_{0}\right)^{2} / t_{0}^{2}\right)\right)}}{\sqrt{2 \pi} \sigma\left(1+\left(t-t_{0}\right)^{2} / t_{0}^{2}\right)^{1 / 2}} \approx \frac{t_{0} e^{-t_{0}^{2} x^{2} /\left(2 \sigma^{2} t^{2}\right)}}{\sqrt{2 \pi} \sigma t}\left(\text { for } t \gg t_{0}\right) \\
& =\frac{t_{0} g\left(t_{0} x / t\right)}{t}
\end{aligned}
$$

where

$$
g(x)=|\psi|^{2}\left(t_{0}, x\right)=\frac{e^{-x^{2} /\left(2 \sigma^{2}\right)}}{\sqrt{2 \pi} \sigma}
$$

Thus, for $\left(y=0\right.$ and) large values of $t$ (relative to $t_{0}$ ) the description of $\rho_{y}$ given in (6) is indeed fully compatible with the quantum-mechanical one found in (8). (Note: a discussion of the potential conceptual significance of the specific choice of $t_{0}$ in (7) can be found in [1].)

To proceed, we define $L_{y}(t, x)$ to be the kinetic energy of a free particle of mass $m$ that travels along the flow line 
of $\underline{\mathrm{v}}_{y}$ through $(t, x)$ with the corresponding flow-line velocity $(x-y) / t$, i.e.,

$$
L_{y}(t, x):=\frac{m}{2}\left(\frac{x-y}{t}\right)^{2} .
$$

Given this definition, it is easy to see that

$$
\psi_{y}(t, x):=\frac{e^{i L_{y}(t, x) t}}{\sqrt{t}}
$$

is a solution of (4) as well of the free-particle Schrödinger equation (in Planck-scale units):

$$
i \frac{\partial \psi}{\partial t}=-\frac{1}{2 m} \frac{\partial^{2} \psi}{\partial x^{2}}
$$

Furthermore, in order to gain the flexibility necessary to adjust the solutions of this equation to a given initial condition, we introduce a function $h: \mathbb{R} \rightarrow \mathbb{C}$, set

$$
\alpha:=\sqrt{\frac{m}{2 \pi i}},
$$

and define a superposition of all the wave functions $\psi_{y}$ via the equation

$$
\psi(t, x)=\int_{-\infty}^{\infty} \alpha h(y) \psi_{y}(t, x) d y=\int_{-\infty}^{\infty} \frac{\alpha h(y) e^{i L_{y}(t, x) t}}{\sqrt{t}} d y .
$$

Recalling further the well known fact that any solution $\psi$ of (9) is of the form

$$
\psi(t, x)=\int_{-\infty}^{\infty} \frac{\alpha \psi(0, y) e^{i L_{y}(t, x) t}}{\sqrt{t}} d y,
$$

it follows that $h(x)=\psi(0, x)$ and that $\psi$, as defined in $(10)$, is the general solution of (9).

\section{Particles in Arbitrary Potential Fields}

In order to generalize the result just established concerning the representability of solutions of (9) by superpositions of solutions of (23), we will consider next a particle of mass $m$ whose motion is constrained by a force field that is generated by a potential function $V: \mathbb{R} \rightarrow \mathbb{R}$. Assuming that $\underline{\mathrm{v}}_{y}$ is well defined for all values $t$ that are less than or equal to some positive constant $T$ (see the pertinent remark in the Introduction), we will now establish the following lemma:

\section{Lemma 1.}

If we denote by $L(x, v)$ the Lagrangian $m v^{2} / 2-V(x)$, then, for any $a \in(0, T)$, it is the case that

$$
\begin{gathered}
\psi_{a, y}(t, x):= \\
\exp \left(\int_{0}^{t} i L\left(z_{y, t, x}(\tau), v_{y}\left(c_{y, t, x}(\tau)\right)\right) d \tau-\frac{1}{2} \int_{a}^{t} \operatorname{div}_{\underline{v}_{y}}\left(c_{y, t, x}(\tau)\right) d \tau\right)
\end{gathered}
$$

is a solution of the equation

$$
\partial_{v_{y}(t, x)} \psi=-\frac{\psi}{2} \operatorname{div}_{\underline{v}_{y}}(t, x)+i L\left(x, v_{y}(t, x)\right) \psi
$$

on the set $(0, T) \times \mathbb{R}$ and that

$$
\begin{aligned}
i \frac{\partial \psi_{a, y}}{\partial t}= & -\frac{1}{2 m} \frac{\partial^{2} \psi_{a, y}}{\partial x^{2}}+V(x) \psi_{a, y} \\
& -\frac{\psi_{a, y}}{4 m} \int_{a}^{t} \frac{\partial^{2}}{\partial x^{2}} \operatorname{div} v_{y} d \tau+\frac{\psi_{a, y}}{8 m}\left(\int_{a}^{t} \frac{\partial}{\partial x} \operatorname{div} v_{y} d \tau\right)^{2} .
\end{aligned}
$$

Proof. Since $c_{y, t, x}^{\prime}(t)=\underline{\mathrm{v}}_{y}(t, x)$, and since evidently

$$
c_{y, t, x}(\tau)=c_{y, c_{y, t, x}(s)}(\tau)
$$

for all $s$ and $\tau$ in the domain of $c_{y, t, x}$, it follows that

$$
\begin{aligned}
\partial_{v_{y}(t, x)} \psi_{a, y}= & \left.\frac{d}{d s}\right|_{s=t} \psi_{a, y}\left(c_{y, t, x}(s)\right) \\
= & \left.\frac{d}{d s}\right|_{s=t} \exp \left(\int_{0}^{s} i L\left(z_{y, t, t}(\tau), v_{y}\left(c_{y, t, x}(\tau)\right)\right), d \tau\right. \\
& \left.-\frac{1}{2} \int_{a}^{s} \operatorname{div} v_{y}\left(c_{y, t, x}(\tau)\right), d \tau\right) .
\end{aligned}
$$

Hence $\psi_{a, y}$ is a solution of (12).

In order to prove that $\psi_{a, y}$ satisfies (13) as well, we use the equation of motion

$$
\frac{d}{d \tau} \frac{\partial L}{\partial v}=\frac{\partial L}{\partial x}
$$

to infer that

$$
\begin{aligned}
\frac{\partial \psi_{a, y}}{\partial t}= & -\frac{\psi_{a, y}}{2} \operatorname{div}_{\underline{v}_{y}}(t, x)-\frac{\psi_{a, y}}{2} \int_{a}^{t} \frac{\partial}{\partial t} \operatorname{div}_{\underline{v}_{y}}\left(c_{y, t, x}(\tau)\right) d \tau \\
& +i L\left(x, v_{y}(t, x)\right) \psi_{a, y}+i \psi_{a, y} \int_{0}^{t} \frac{\partial}{\partial t} L\left(z_{y, t, x}(\tau), v_{y}\left(c_{y, t, x}(\tau)\right)\right) d \tau \\
= & -\frac{\psi_{a, y}}{2} \frac{\partial v_{y}}{\partial x}-\frac{\psi_{a, y}}{2} \int_{a}^{t} \frac{\partial}{\partial t} \operatorname{div} \underline{v}_{y} d \tau+i L \psi_{a, y} \\
& +i \psi_{a, y} \int_{0}^{t}\left(\left(\frac{d}{d \tau} \frac{\partial L}{\partial v}\right) \frac{\partial z_{y, t, x}(\tau)}{\partial t}+\frac{\partial L}{\partial v} \frac{\partial v_{y}\left(c_{y, t, x}(\tau)\right)}{\partial t}\right) d \tau \\
= & -\frac{\psi_{a, y}}{2} \frac{\partial v_{y}}{\partial x}-\frac{\psi_{a, y}}{2} \int_{a}^{t} \frac{\partial}{\partial t} \operatorname{div} v_{y} d \tau+i \psi_{a, y}\left(\left.\frac{\partial L}{\partial v} \frac{\partial z_{y, t}, x}{\partial t}(\tau)\right|_{0} ^{t}\right) \\
& +i L \psi_{a, y}+i \psi_{a, y} \int_{0}^{t}\left(-\frac{\partial L}{\partial v} \frac{\partial z_{y, t, x}^{\prime}(\tau)}{\partial t}+\frac{\partial L}{\partial v} \frac{\partial v_{y}\left(c_{y, t, x}(\tau)\right)}{\partial t}\right) d \tau \\
= & -\frac{\psi_{a, y}}{2} \frac{\partial v_{y}}{\partial x}-\frac{\psi_{a, y}}{2} \int_{a}^{t} \frac{\partial}{\partial t} \operatorname{div} \underline{v}_{y} d \tau+i L \psi_{a, y} \\
& +i \psi_{a, y}\left(\left.\frac{\partial L}{\partial v} \frac{\partial z_{y, t, x}(\tau)}{\partial t}\right|_{\tau=t}-\frac{\partial L}{\partial v} \frac{\partial y}{\partial t}\right) \\
& +i \psi_{a, y} \int_{0}^{t}\left(-\frac{\partial L}{\partial v} \frac{\partial v_{y}\left(c_{y, t, x}(\tau)\right)}{\partial t}+\frac{\partial L}{\partial v} \frac{\partial v_{y}\left(c_{y, t, x}(\tau)\right)}{\partial t}\right) d \tau \\
= & \frac{\psi_{a, y}}{2}\left(-\frac{\partial v_{y}}{\partial x}-\int_{a}^{t} \frac{\partial}{\partial t} \operatorname{div} \underline{v}_{y} d \tau+2 i L+\left.2 i \frac{\partial L}{\partial v} \frac{\partial z_{y, t, x}(\tau)}{\partial t}\right|_{\tau=t}\right) \\
= & \frac{\psi_{a, y}}{2}\left(-\frac{\partial v_{y}}{\partial x}-\int_{a}^{t} \frac{\partial}{\partial t} \operatorname{div}_{\underline{v}_{y}} d \tau+2 i L+\left.2 i m v_{y} \frac{\partial z_{y, t, x}(\tau)}{\partial t}\right|_{\tau=t}\right) .
\end{aligned}
$$


Similarly, we find that

$$
\begin{aligned}
\frac{\partial^{2} \psi_{a, y}}{\partial x^{2}}= & -\frac{\partial}{\partial x}\left(\frac{\psi_{a, y}}{2} \int_{a}^{t} \frac{\partial}{\partial x} \operatorname{div} \underline{\mathbf{v}}_{y}\left(c_{y, t, x}(\tau)\right) d \tau\right) \\
& +\frac{\partial}{\partial x}\left(i \psi_{a, y} \int_{0}^{t} \frac{\partial}{\partial x} L\left(z_{y, t, x}(\tau), v_{y}\left(c_{y, t, x}(\tau)\right)\right) d \tau\right) \\
= & \frac{\partial}{\partial x}\left(\left(-\frac{1}{2} \int_{a}^{t} \frac{\partial}{\partial x} \operatorname{div} \underline{\underline{v}}_{y} d \tau+i m v_{y}\left(\left.\frac{\partial z_{y, t, x}(\tau)}{\partial x}\right|_{\tau=t}\right)\right) \psi_{a, y}\right) \\
= & \frac{\partial}{\partial x}\left(\left(-\frac{1}{2} \int_{a}^{t} \frac{\partial}{\partial x} \operatorname{div} v_{y} d \tau+i m v_{y} \frac{\partial x}{\partial x}\right) \psi_{a, y}\right) \\
= & \left(-\frac{1}{2} \int_{a}^{t} \frac{\partial}{\partial x} \operatorname{div} \underline{\mathbf{v}}_{y} d \tau+i m v_{y}\right)^{2} \psi_{a, y} \\
& +\psi_{a, y} \frac{\partial}{\partial x}\left(-\frac{1}{2} \int_{a}^{t} \frac{\partial}{\partial x} \operatorname{div} \underline{\underline{v}}_{y} d \tau+i m v_{y}\right) \\
= & -m^{2} \psi_{a, y} v_{y}^{2}+i m \psi_{a, y} \frac{\partial v_{y}}{\partial x}-i m \psi_{a, y} v_{y} \int_{a}^{t} \frac{\partial}{\partial x} \operatorname{div} \underline{\mathbf{v}}_{y} d \tau \\
& +\frac{\psi_{a, y}}{4}\left(\int_{a}^{t} \frac{\partial}{\partial x} \operatorname{div} \underline{\mathbf{v}}_{y} d \tau\right)^{2}-\frac{\psi_{a, y}}{2} \int_{a}^{t} \frac{\partial^{2}}{\partial x^{2}} \operatorname{div} \underline{\mathbf{v}}_{y} d \tau .
\end{aligned}
$$

Finally, according to (14), we have

$$
\begin{aligned}
0 & =\left.\frac{d}{d s} z_{y, c_{y, x, t}(s)}(\tau)\right|_{(s, \tau)=(t, t)} \\
& =\left.\frac{\partial z_{y, t, x}(\tau)}{\partial t}\right|_{\tau=t}+\left.v_{y} \frac{\partial z_{y, t, x}(\tau)}{\partial x}\right|_{\tau=t} \\
& =\left.\frac{\partial z_{y, t, x}(\tau)}{\partial t}\right|_{\tau=t}+v_{y},
\end{aligned}
$$

and

$$
\begin{aligned}
0 & =\left.\frac{d}{d s}\right|_{s=t} \operatorname{div} \underline{\mathrm{v}}_{y}\left(c_{y, c_{y, t, x}(s)}(\tau)\right)=\partial_{\underline{\mathbf{v}}_{y}(t, x)} \operatorname{div} \underline{\mathrm{v}}_{y}\left(c_{y, t, x}(\tau)\right) \\
& =\frac{\partial}{\partial t} \operatorname{div} \underline{\mathbf{v}}_{y}\left(c_{y, t, x}(\tau)\right)+v_{y}(t, x) \frac{\partial}{\partial x} \operatorname{div}_{\underline{\mathrm{v}}_{y}}\left(c_{y, t, x}(\tau)\right),
\end{aligned}
$$

and therefore,

$$
\begin{aligned}
i \frac{\partial \psi_{a, y}}{\partial t}= & -\frac{1}{2 m} \frac{\partial^{2} \psi_{a, y}}{\partial x^{2}}+V(x) \psi_{a, y} \\
& -\frac{\psi_{a, y}}{4 m} \int_{a}^{t} \frac{\partial^{2}}{\partial x^{2}} \operatorname{div} \underline{v}_{y} d \tau \\
& +\frac{\psi_{a, y}}{8 m}\left(\int_{a}^{t} \frac{\partial}{\partial x} \operatorname{div}_{\underline{v}_{y}} d \tau\right)^{2}
\end{aligned}
$$

as desired. q.e.d.

\section{Proposition 2.}

If $V(x)=m \omega^{2} x^{2} / 2$ is a harmonic oscillator potential, then $\psi_{a, y}$ is a solution of the Schrödinger equation

$$
i \frac{\partial \psi}{\partial t}=-\frac{1}{2 m} \frac{\partial^{2} \psi}{\partial x^{2}}+V(x) \psi
$$

for all $a \in(0, \pi / \omega)$.
Proof. Given the equation of motion

$$
z_{y, t, x}^{\prime \prime}(s)+\frac{1}{m} V^{\prime}\left(z_{y, t, x}(s)\right)=z_{y, t, x}^{\prime \prime}(s)+\omega^{2} z_{y, t, x}(s)=0,
$$

it readily follows that

$$
\begin{aligned}
& z_{y, t, x}(s)=y \cos (\omega s)+\frac{(x-y \cos (\omega t)) \sin (\omega s)}{\sin (\omega t)} \\
& \underline{\mathrm{v}}_{y}(t, x)=\left(\begin{array}{c}
1 \\
\omega(x \cos (\omega t)-y) / \sin (\omega t)
\end{array}\right)
\end{aligned}
$$

and

$$
\operatorname{div} \underline{\mathrm{v}}_{y}(t, x)=\omega \cot (\omega t)
$$

This yields

$$
\frac{\partial}{\partial x} \operatorname{div} \underline{\mathrm{v}}_{y}(t, x)=0,
$$

and therefore, the integrals in (13) are both equal to zero. q.e.d.

Remark 2If $V(\mathrm{x})=(\mathrm{m} / 2) \mathrm{x}^{\mathrm{t}} \Omega \mathrm{x}$ is a harmonic oscillator potential on $\mathbb{R}^{3}$ (for some positive definite, symmetric $3 \times 3$ matrix $\Omega$ ), then the proof of Proposition 2 can be given in exactly the same way as above as long as the corresponding spatial coordinates $x^{1}, x^{2}$, and $x^{3}$ are understood to refer to an orthonormal basis of eigenvectors of $\Omega$. For given this choice of the coordinate system, equation (15) is easily seen to remain valid for $x^{1}$, $x^{2}$, and $x^{3}$. That is to say, $\partial / \partial x^{i} \operatorname{div} \underline{\mathrm{v}}_{y}(t, \mathrm{x})=0$ for all $i \in\{1,2,3\}$ whenever $V$ is a second-order polynomial potential.

Remark 3The parameter $T$ that is referred to in the statement of Lemma 1 is equal to $\pi / \omega$ in the case of a harmonic oscillator potential because both $z_{y, t, x}$ and $v_{y}$ have singularities at $t=\pi / \omega$.

To proceed, we observe that a superposition analogous to (10) is

$$
\psi(t, x)=\int_{-\infty}^{\infty} \alpha h(y) \psi_{a, y}(t, x) d y .
$$

Using elementary analytical arguments, it is not difficult to show that for $a:=\pi /(2 \omega)$ it is the case that

$$
\lim _{t \rightarrow 0^{+}} \psi(t, x)=\frac{h(x)}{\sqrt{\omega}} .
$$

This shows that the initial state $\psi(0, x)$ is essentially equal to $h(x)$ and that therefore the full set of solutions of the Schrödinger equation can be generated via superpositions of solutions of equation (4), whenever $V$ is a harmonic oscillator potential. (Note: the presence of the factor $1 / \sqrt{\omega}$ in the limit above is due to the fact that we chose $a$ to be equal to $\pi /(2 \omega)$. If, instead, we had defined 
$a$ to be one-which is feasible only if $\omega<\pi$-this factor would not have appeared.)

We will forego a detailed proof of equation (17) because in preparation for our subsequent discussion of path-integration, we will now examine the right-hand limit of $\psi$ at $t=0$ in the most general setting when the potential $V$ is arbitrary (up to certain regularity conditions that will facilitate our pertinent calculations). To this end, we notice to begin with that

$$
\begin{aligned}
\frac{d}{d \tau} \frac{\partial z_{y, t, x}(\tau)}{\partial x} & =\frac{\partial z_{y, t, x}^{\prime}(\tau)}{\partial x}=\frac{\partial}{\partial x} v_{y}\left(c_{y, t, x}(\tau)\right) \\
& =\left.\frac{\partial v_{y}}{\partial x}\right|_{c_{y, t, x}(\tau)} \frac{\partial z_{y, t, x}(\tau)}{\partial x} \\
& =\operatorname{div}_{\underline{v}_{y}}\left(c_{y, t, x}(\tau)\right) \frac{\partial z_{y, t, x}(\tau)}{\partial x}
\end{aligned}
$$

This yields

$$
\operatorname{div} \underline{\mathrm{v}}_{y}\left(c_{y, t, x}(\tau)\right)=\frac{d}{d \tau} \ln \left|\frac{\partial z_{y, t, x}(\tau)}{\partial x}\right|
$$

and, by implication,

$$
\begin{aligned}
\psi_{a, y}(t, x) & =\frac{\sqrt{\left|\partial z_{y, t, x}(a) / \partial x\right|}}{\sqrt{\left|\partial z_{y, t, x}(t) / \partial x\right|}} \exp \left(\int_{0}^{t} i L\left(z_{y, t, x}(\tau), v_{y}\left(c_{y, t, x}(\tau)\right)\right) d \tau\right) \\
& =\sqrt{\left|\frac{\partial z_{y, t, x}(a)}{\partial x}\right|} \exp \left(\int_{0}^{t} i L\left(z_{y, t, x}(\tau), v_{y}\left(c_{y, t, x}(\tau)\right)\right) d \tau\right)
\end{aligned}
$$

because

$$
\frac{\partial z_{y, t, x}(t)}{\partial x}=\frac{\partial x}{\partial x}=1
$$

Given this result, we now move on to normalize equation (16) by eliminating the dependence of $\psi$ on $a$. To do so, we define

$$
\psi_{y}(t, x):=\lim _{a \rightarrow 0^{+}} \frac{\psi_{a, y}(t, x)}{\sqrt{a}} .
$$

\section{Proposition 3.}

For all $(t, x) \in(0, T) \times \mathbb{R}$ it is the case that $\psi_{y}(t, x)=\sqrt{\left|\frac{\partial z_{y, t, x}^{\prime}(0)}{\partial x}\right|} \exp \left(\int_{0}^{t} i L\left(z_{y, t, x}(\tau), v_{y}\left(c_{y, t, x}(\tau)\right)\right) d \tau\right)$.

Proof. Using the representation of $\psi_{a, y}$ in (18) and the boundary condition $z_{y, t, x}(0)=y$, it follows that

$$
\begin{gathered}
\lim _{a \rightarrow 0^{+}} \frac{\partial z_{y, t, x}(a) / \partial x}{a}= \\
\lim _{a \rightarrow 0^{+}} \frac{\partial z_{y, t, x}(a) / \partial x-\partial z_{y, t, x}(0) / \partial x}{a}=\frac{\partial z_{y, t, x}^{\prime}(0)}{\partial x},
\end{gathered}
$$

as desired. q.e.d.
In order to show that the initial state of the superposition

$$
\psi(t, x)=\int_{-\infty}^{\infty} \alpha h(y) \psi_{y}(t, x) d y
$$

is equal to $h(x)$, we first observe that

$$
\begin{aligned}
z_{y, t, x}(\tau) & =z_{y, t, x}(0)+\int_{0}^{\tau} z_{y, t, x}^{\prime}(\sigma) d \sigma \\
& =z_{y, t, x}(0)+z_{y, t, x}^{\prime}(0) \tau+\int_{0}^{\tau} \int_{0}^{\sigma} z_{y, t, x}^{\prime \prime}(\rho) d \rho d \sigma \\
& =z_{y, t, x}(0)+z_{y, t, x}^{\prime}(0) \tau-\frac{1}{m} \int_{0}^{\tau} \int_{\rho}^{\tau} V^{\prime}\left(z_{y, t, x}(\rho)\right) d \sigma d \rho \\
& =z_{y, t, x}(0)+z_{y, t, x}^{\prime}(0) \tau-\frac{1}{m} \int_{0}^{\tau}(\tau-\rho) V^{\prime}\left(z_{y, t, x}(\rho)\right) d \rho
\end{aligned}
$$

Setting $\tau=t$, it follows that

$$
x=y+z_{y, t, x}^{\prime}(0) t-\frac{1}{m} \int_{0}^{t}(t-\rho) V^{\prime}\left(z_{y, t, x}(\rho)\right) d \rho
$$

and therefore

$$
z_{y, t, x}^{\prime}(0)=\frac{x-y}{t}+\frac{1}{m t} \int_{0}^{t}(t-\rho) V^{\prime}\left(z_{y, t, x}(\rho)\right) d \rho
$$

Hence

$\frac{\partial z_{y, t, x}^{\prime}(0)}{\partial x}=\frac{1}{t}\left(1+\frac{1}{m} \int_{0}^{t}(t-\rho) V^{\prime \prime}\left(z_{y, t, x}(\rho)\right) \frac{\partial z_{y, t, x}(\rho)}{\partial x} d \rho\right)$.

Since

$$
\frac{\partial z_{y, t, x}(0)}{\partial x}=\frac{\partial y}{\partial x}=0
$$

and

$$
\frac{\partial z_{y, t, x}(t)}{\partial x}=\frac{\partial x}{\partial x}=1
$$

it is eminently plausible to assume that there is a constant $M>1$ such that

$$
\left|\frac{\partial z_{y, t, x}(\rho)}{\partial x}\right| \leq M
$$

for all $x, y \in \mathbb{R}$, all $t \in(0, T)$, and all $\rho \in[0, t]$. (Note: a proof of the existence of $M$ under adequate regularity assumptions on $V$ can certainly be given but will here be omitted as it would be of very little interest from a physical point of view.) Imposing the additional regularity assumption that $V^{\prime \prime}$ be bounded (i.e., $\left|V^{\prime \prime}\right| \leq M$ ), we may infer that the function

$$
p_{t, x}(y):=\sqrt{\left|1+\frac{1}{m} \int_{0}^{t}(t-\rho) V^{\prime \prime}\left(z_{y, t, x}(\rho)\right) \frac{\partial z_{y, t, x}(\rho)}{\partial x} d \rho\right|}
$$

satisfies the estimate

$$
\left|p_{t, x}(y)-1\right|=\frac{\left|p_{t, x}(y)^{2}-1\right|}{\left|p_{t, x}(y)+1\right|} \leq\left|p_{t, x}(y)^{2}-1\right| \leq \frac{M^{2} t^{2}}{2 m}
$$


for all $x \in \mathbb{R}$ and all $t \in(0, T)$. To proceed we notice that

$$
v_{y}\left(c_{y, t, x}(\tau)\right)=z_{y, t, x}^{\prime}(\tau)=z_{y, t, x}^{\prime}(0)-\frac{1}{m} \int_{0}^{\tau} V^{\prime}\left(z_{y, t, x}(\sigma)\right) d \sigma
$$

and set

$$
\begin{aligned}
q_{t, x}(y):= & \int_{0}^{t} \frac{1}{2 m}\left(\frac{1}{t^{2}}\left(\int_{0}^{t}(t-\rho) V^{\prime} d \rho\right)^{2}+\left(\int_{0}^{\tau} V^{\prime} d \sigma\right)^{2}\right) d \tau \\
& -\int_{0}^{t}\left(\frac{1}{m t} \int_{0}^{t}(t-\rho) V^{\prime} d \rho \int_{0}^{\tau} V^{\prime} d \sigma+V\right) d \tau
\end{aligned}
$$

and

$$
r_{t, x}(y):=\int_{0}^{t} \frac{1}{t}\left(\frac{1}{t} \int_{0}^{t}(t-\rho) V^{\prime} d \rho-\int_{0}^{\tau} V^{\prime} d \sigma\right) d \tau
$$

Combining Proposition 3 with (19) and (20), it is easy to see that

$$
\begin{gathered}
\psi_{y}(t, x)= \\
\frac{p_{t, x}(y)}{\sqrt{t}} \exp \left(\frac{i m(x-y)^{2}}{2 t}+i(x-y) r_{t, x}(y)+i q_{t, x}(y)\right) .
\end{gathered}
$$

In order to accomplish our present objective-namely the determination of the initial state of the superposition $\psi$-it is natural and convenient to further assume that $V$ and $V^{\prime}$ be bounded as well (i.e., $|V|,\left|V^{\prime}\right| \leq M$ ). Then

$$
\begin{aligned}
& \left|\exp \left(i q_{t, x}(y)\right)-1\right|=2\left(1-\cos \left(q_{t, x}(y)\right)\right) \leq q_{t, x}(y)^{2} \\
& \leq\left(\int_{0}^{t}\left(\frac{M^{2} t^{2}}{8 m}+\frac{M^{2} \tau^{2}}{2 m}+\frac{M^{2} \tau t}{2 m}+M\right) d \tau\right)^{2} \\
& =M^{2} t^{2}\left(\frac{13 M t^{2}}{24 m}+1\right)^{2}, \\
& \text { and } \\
& \left|\int_{-\infty}^{\infty} \frac{h(y)\left(p_{t, x}(y) \exp \left(i q_{t, x}(y)\right)-1\right)}{\sqrt{t}} \exp \left(\frac{i m(x-y)^{2}}{2 t}+i(x-y) r_{t, x}(y)\right) d y\right| \\
& \leq \int_{-\infty}^{\infty} \frac{|h(y)|\left|p_{t, x}(y) \exp \left(i q_{t, x}(y)\right)-1\right|}{\sqrt{t}} d y \\
& \leq \int_{-\infty}^{\infty} \frac{|h(y)|\left(\left|p_{t, x}(y)-1\right|+\left|\exp \left(i q_{t, x}(y)\right)-1\right|\right)}{\sqrt{t}} d y \\
& \leq M^{2} t \sqrt{t}\left(\frac{1}{2 m}+\left(\frac{13 M t^{2}}{24 m}+1\right)^{2}\right) \int_{-\infty}^{\infty}|h(y)| d y \quad \text { (by (22) and (25)). }
\end{aligned}
$$

Since the latter term vanishes at $t=0$, it follows that

$$
\begin{gathered}
\lim _{t \rightarrow 0^{+}} \psi(t, x)= \\
\lim _{t \rightarrow 0^{+}} \int_{-\infty}^{\infty} \frac{\alpha h(y)}{\sqrt{t}} \exp \left(\frac{i m(x-y)^{2}}{2 t}+i(x-y) r_{t, x}(y)\right) d y .
\end{gathered}
$$

According to (11), we have

$$
h(x)=\lim _{t \rightarrow 0^{+}} \int_{-\infty}^{\infty} \frac{\alpha h(y)}{\sqrt{t}} \exp \left(\frac{i m(x-y)^{2}}{2 t}\right) d y
$$

and therefore

$$
\begin{aligned}
\lim _{t \rightarrow 0^{+}}|\psi(t, x)-h(x)| & \leq \lim _{t \rightarrow 0^{+}} \int_{-\infty}^{\infty} \frac{|\alpha h(y)|}{\sqrt{t}}\left|\exp \left(i(x-y) r_{t, x}(y)\right)-1\right| d y \\
& =\lim _{t \rightarrow 0^{+}} \int_{-\infty}^{\infty} \frac{|2 \alpha h(y)|}{\sqrt{t}}\left(1-\cos \left((x-y) r_{t, x}(y)\right)\right) d y \\
& \leq \lim _{t \rightarrow 0^{+}} \int_{-\infty}^{\infty} \frac{|\alpha h(y)|}{\sqrt{t}}(x-y)^{2} r_{t, x}(y)^{2} d y .
\end{aligned}
$$

Using the definition of $r_{t, x}(y)$ in (24), we find that

$$
r_{t, x}(y)^{2} \leq\left(\int_{0}^{t}\left(\frac{M}{2}+\frac{M \tau}{t}\right) d \tau\right)^{2}=M^{2} t^{2},
$$

and, by implication,

$\lim _{t \rightarrow 0^{+}}|\psi(t, x)-h(x)| \leq \lim _{t \rightarrow 0^{+}} M^{2} t \sqrt{t} \int_{-\infty}^{\infty}|\alpha h(y)|(x-y)^{2} d y$.

Since $h(y)(x-y)^{2}$ may clearly be assumed to be integrable, we finally arrive at the desired conclusion:

$$
\lim _{t \rightarrow 0^{+}} \psi(t, x)=h(x)
$$

Having established this equality, our next goal is to introduce a path-integral formalism that generates the full set of solutions of the Schrödinger equation for a potential function $V$ that satisfies the various regularity conditions imposed in the preceding computations but is otherwise essentially arbitrary. Picking a (large) $n \in \mathbb{N}$ and a $b \in(0, T)$, we set $t_{k}:=k b / 2^{n}$ and $I_{k}:=\left(t_{k-1}, t_{k}\right]$ for all $k \in\left\{1, \ldots, 2^{n}\right\}$. Moreover, for a given $t \in I_{k}$ we denote by $z_{k, y, t, x}$ the spatial coordinate of a particle of mass $m$ that moves in the force field generated by $V$ and satisfies the boundary conditions $z_{k, y, t, x}\left(t_{k-1}\right)=y$ and $z_{k, y, t, x}(t)=x$. The analogous defining equations for $\underline{\mathrm{v}}_{k, y}$ and $c_{k, y, t, x}$ are

$$
\underline{\mathrm{v}}_{k, y}(t, x)=\left(\begin{array}{c}
1 \\
v_{k, y}(t, x)
\end{array}\right):=\left(\begin{array}{c}
1 \\
z_{k, y, t, x}^{\prime}(t)
\end{array}\right)
$$

and

$$
c_{k, y, t, x}(s):=\left(\begin{array}{c}
s \\
z_{k, y, t, x}(s)
\end{array}\right) .
$$

Making direct reference to the result stated in Proposition 3, we further define

$$
\begin{gathered}
\psi_{k, y}(t, x):=\sqrt{\left|\frac{\partial z_{k, y, t, x}\left(t_{k-1}\right)}{\partial x}\right|} \\
\exp \left(\int_{t_{k-1}}^{t} i L\left(z_{k, y, t, x}(\tau), v_{k, y}\left(c_{k, y, t, x}(\tau)\right)\right) d \tau\right)
\end{gathered}
$$

for all $(t, x) \in I_{k} \times \mathbb{R}$, and, proceeding recursively, we set

$$
\psi_{1}(t, x):=\int_{-\infty}^{\infty} \alpha h(y) \psi_{1, y}(t, x) d y
$$

and

$$
\psi_{k+1}(t, x):=\int_{-\infty}^{\infty} \alpha \psi_{k}\left(t_{k}, y\right) \psi_{k+1, y}(t, x) d y,
$$


where the domain of each $\psi_{k}$ is understood to be $I_{k} \times \mathbb{R}$. Thus, the $n$-th approximation to the desired path-integral superposition $\psi$ is the piecewise function $\psi^{n}:(0, b] \times \mathbb{R} \rightarrow \mathbb{C}$ which, by definition, equals $\psi_{k}$ on $I_{k}$ for each $k \in\left\{1, \ldots, 2^{n}\right\}$. (Note: the continuity of $\psi^{n}$ is crucially guaranteed by (26).) Given the recursive pattern defined in (27) and (28), it is easy to see that for any $k \in\left\{1, \ldots, 2^{n}\right\}$ and any $t \in I_{k}$ we have $\psi^{n}(t, x)=\alpha^{k} \int_{\mathbb{R}^{k}} h\left(y_{1}\right)\left(\prod_{j=1}^{k-1} \psi_{j, y_{j}}\left(t_{j}, y_{j+1}\right)\right) \psi_{k, y_{k}}(t, x) d y_{1} \cdots d y_{k}$

As is customary in discussions of path-integral formalisms, we will omit a rigorous justification for the existence of the limit

$$
\psi(t, x):=\lim _{n \rightarrow \infty} \psi^{n}(t, x)
$$

and focus our attention instead on explaining why $\psi$ can plausibly be regarded as a solution of the initial value problem

$$
i \frac{\partial \psi}{\partial t}=-\frac{1}{2 m} \frac{\partial^{2} \psi}{\partial x^{2}}+V(x) \psi
$$

and

$$
\psi(0, x)=h(x)
$$

To this end, we pick an arbitrary $t \in(0, b]$ and a corresponding $k \in\left\{1, \ldots, 2^{n}\right\}$ such that $t \in I_{k}$. If $n$ is large, then

$$
\psi\left\lceil_ { I _ { k } \times \mathbb { R } } \approx \psi ^ { n } \left\lceil_{I_{k} \times \mathbb{R}}=\psi_{k},\right.\right.
$$

and Lemma 1 in conjunction with Proposition 3 therefore implies that for all $a \in I_{k}$ that are sufficiently close to $t_{k-1}$ it is the case that

$$
\begin{array}{cc}
i \frac{\partial \psi}{\partial t} \approx & -\frac{1}{2 m} \frac{\partial^{2} \psi}{\partial x^{2}}+V(x) \psi \\
& +\int_{-\infty}^{\infty} \alpha \psi_{k-1}\left(t_{k-1}, y\right) \psi_{k, y}(t, x) G_{k, a, y}(t, x) d y
\end{array}
$$

where

$G_{k, a, y}(t, x):=\frac{1}{8 m}\left(\int_{a}^{t} \frac{\partial}{\partial x} \operatorname{div} \underline{\mathrm{v}}_{k, y} d \tau\right)^{2}-\frac{1}{4 m} \int_{a}^{t} \frac{\partial^{2}}{\partial x^{2}} \operatorname{div} \underline{\mathrm{v}}_{k, y} d \tau$

Using (19) and (23), it follows that

$$
\begin{aligned}
v_{k, y}(t, x)=z_{k, y, t, x}^{\prime}(t) & =z_{k, y, t, x}^{\prime}\left(t_{k-1}\right) \\
& -\frac{1}{m} \int_{t_{k-1}}^{t} V^{\prime}\left(z_{k, y, t, x}(\sigma)\right) d \sigma \\
& =\frac{x-y}{t-t_{k-1}} \\
& +\int_{t_{k-1}}^{t} \frac{(t-\rho) V^{\prime}\left(z_{k, y, t, x}(\rho)\right)}{m\left(t-t_{k-1}\right)} d \rho \\
& -\frac{1}{m} \int_{t_{k-1}}^{t} V^{\prime}\left(z_{k, y, t, x}(\sigma)\right) d \sigma,
\end{aligned}
$$

and therefore,

$$
\begin{aligned}
\operatorname{div} \underline{\mathrm{v}}_{k, y}(t, x) & =\frac{\partial v_{k, y}}{\partial x}(t, x) \\
& =\frac{1}{t-t_{k-1}} \\
& -\int_{t_{k-1}}^{t} \frac{\left(\rho-t_{k-1}\right) V^{\prime \prime}\left(z_{k, y, t, x}(\rho)\right)}{m\left(t-t_{k-1}\right)} \frac{\partial z_{k, y, t, x}(\rho)}{\partial x} d \rho .
\end{aligned}
$$

Hence

$$
\begin{aligned}
& \operatorname{div} \underline{\underline{v}}_{k, y}\left(c_{k, y, t, x}(\tau)\right)= \\
& \frac{1}{\tau-t_{k-1}} \\
& -\left.\int_{t_{k-1}}^{\tau} \frac{\left(\rho-t_{k-1}\right) V^{\prime \prime}\left(z_{k, y, c_{k, y, t}, x}(\tau)\right)}{m\left(\tau-t_{k-1}\right)} \frac{\partial z_{k, y, \tau, x}(\rho)}{\partial x}\right|_{x=z_{k, y, t, x}(\tau)} d \rho \\
& =\frac{1}{\tau-t_{k-1}} \\
& -\int_{t_{k-1}}^{\tau} \frac{\left(\rho-t_{k-1}\right) V^{\prime \prime}\left(z_{k, y, t, x}(\rho)\right)}{m\left(\tau-t_{k-1}\right)} \frac{\left.\partial z_{k, y, \tau}, \tau, z\right)}{\partial x} d \rho \quad \text { (by (14)) }
\end{aligned}
$$

and

$$
\begin{aligned}
& \frac{\partial}{\partial x} \operatorname{div} \underline{\mathbf{v}}_{k, y}\left(c_{k, y, t, x}(\tau)\right)= \\
& -\int_{t_{k-1}}^{\tau} \frac{\left(\rho-t_{k-1}\right) V^{\prime \prime \prime}\left(z_{k, y, \tau, x}(\rho)\right)}{m\left(\tau-t_{k-1}\right)} \frac{\partial z_{k, y, t, x}(\rho)}{\partial x} \frac{\partial z_{k, y, \tau}, z}{\partial x} d \rho \\
& -\int_{t_{k-1}}^{\tau} \frac{\left(\rho-t_{k-1}\right) V^{\prime \prime}\left(z_{k, y, t, x}(\rho)\right)}{m\left(\tau-t_{k-1}\right)} \frac{\partial^{2} z_{k, y, \tau}(\rho)}{\partial x^{2}} \frac{\partial z_{k, y, t, x}(\tau)}{\partial x} d \rho
\end{aligned}
$$

and

$$
\begin{aligned}
& \frac{\partial^{2}}{\partial x^{2}} \operatorname{div} \underline{v}_{k, y}\left(c_{k, y, t, x}(\tau)\right)= \\
& -\int_{t_{k-1}}^{\tau} \frac{\left(\rho-t_{k-1}\right) V^{(4)}\left(z_{k, y, \tau, x}(\rho)\right)}{m\left(\tau-t_{k-1}\right)}\left(\frac{\partial z_{k, y, t, x}(\rho)}{\partial x}\right)^{2} \frac{\partial z_{k, y, \tau}, z}{\partial x} d \rho \\
& -\int_{t_{k-1}}^{\tau} \frac{\left(\rho-t_{k-1}\right) V^{\prime \prime \prime}\left(z_{k, y, \tau}, x\right.}{m\left(\tau-t_{k-1}\right)} \frac{\partial^{2} z_{k, y, t, x}(\rho)}{\partial x^{2}} \frac{\partial z_{k, y, \tau, z}(\rho)}{\partial x} d \rho \\
& -2 \int_{t_{k-1}}^{\tau} \frac{\left(\rho-t_{k-1}\right) V^{\prime \prime \prime}\left(z_{k, y, \tau, x}(\rho)\right)}{m\left(\tau-t_{k-1}\right)} \frac{\partial z_{k, y, t, x}(\rho)}{\partial x} \frac{\partial^{2} z_{k, y, \tau, z}(\rho)}{\partial x^{2}} \frac{\partial z_{k, y, t, x}(\tau)}{\partial x} d \rho \\
& -\int_{t_{k-1}}^{\tau} \frac{\left(\rho-t_{k-1}\right) V^{\prime \prime}\left(z_{k, y, t, x}(\rho)\right)}{m\left(\tau-t_{k-1}\right)} \frac{\partial^{3} z_{k, y, \tau, z}(\rho)}{\partial x^{3}}\left(\frac{\partial z_{k, y, t, x}(\tau)}{\partial x}\right)^{2} d \rho \\
& -\int_{t_{k-1}}^{\tau} \frac{\left(\rho-t_{k-1}\right) V^{\prime \prime}\left(z_{k, y, t, x}(\rho)\right)}{m\left(\tau-t_{k-1}\right)} \frac{\partial^{2} z_{k, y, \tau, z}(\rho)}{\partial x^{2}} \frac{\partial^{2} z_{k, y, t, x}(\tau)}{\partial x^{2}} d \rho
\end{aligned}
$$

In order to estimate the absolute magnitude of these derivatives of the divergence of $\underline{\mathrm{v}}_{k, y}$, we assume that $V^{\prime \prime \prime}$ and $V^{(4)}$ are bounded (i.e., $\left|V^{\prime \prime \prime}\right|,\left|V^{(4)}\right| \leq M$ ), and-in direct analogy to (21) — we also assume that

$$
\left|\frac{\partial^{2} z_{k, y, t, x}(\rho)}{\partial x^{2}}\right|,\left|\frac{\partial^{3} z_{k, y, t, x}(\rho)}{\partial x^{3}}\right| \leq M
$$

for all $x, y \in \mathbb{R}$, all $t \in I_{k}$, and all $\rho \in\left(t_{k-1}, t\right]$. Then we readily find that

$$
\left|\frac{\partial}{\partial x} \operatorname{div}_{\underline{\mathrm{v}}_{k, y}}\left(c_{k, y, t, x}(\tau)\right)\right| \leq \frac{M^{3}\left(\tau-t_{k-1}\right)}{m} \leq \frac{M^{3} b}{m 2^{n}}
$$

and

$$
\left|\frac{\partial^{2}}{\partial x^{2}} \operatorname{div} \underline{\mathrm{v}}_{k, y}\left(c_{k, y, t, x}(\tau)\right)\right| \leq \frac{3 M^{4}\left(\tau-t_{k-1}\right)}{m} \leq \frac{M^{4} b}{m 2^{n-2}} .
$$


Consequently, if $t_{k-1}<a \leq t$, then

$$
\begin{aligned}
\left|G_{k, a, y}(t, x)\right| & \leq \frac{(t-a)^{2} M^{6} b^{2}}{m^{3} 2^{2 n+3}}+\frac{(t-a) M^{4} b}{m^{2} 2^{n}} \\
& <\frac{M^{6} b^{4}}{m^{3} 2^{4 n+3}}+\frac{M^{4} b^{2}}{m^{2} 2^{2 n}},
\end{aligned}
$$

and, by implication,

$$
\lim _{n \rightarrow \infty} \int_{-\infty}^{\infty} \alpha \psi_{k-1}\left(t_{k-1}, y\right) \psi_{k, y}(t, x) G_{k, a, y}(t, x) d y=0
$$

According to (32), this result may be considered a semirigorous derivation of the proposed fact that $\psi$, as defined in (29), is a solution of the Schrödinger equation (as given in (30)). Furthermore, since $\psi^{n}(t, x)=\psi_{1}(t, x)$ for all $n \in$ $\mathbb{N}$ and all $t \in I_{1}$, the equations (26) and (27) together imply that $\psi$ satisfies the initial condition given in (31). Thus it does appear correct to assert that the totality of solutions of the Schrödinger equation can be constructed from pathintegral superpositions of solutions of equation (4).

\section{References}

[1] Blume, Frank, How Lightlike Motion Links General Relativity to the Path-Integral Formalism of Quantum Mechanics, (available upon request).

[2] Flügge, Siegfried, Practical Quantum Mechanics, Springer, Berlin, (1999).

[3] Kleinert, Hagen, Path Integrals in Quantum Mechanics, Statistics, Polymer Physics, and Financial Markets, 4th Edition, World Scientific, New Jersey, (2006). 